\title{
Ventilation Method Assessment of the Average Permeability of the Fractured Granite Rock Mass around Tunnel
}

\author{
Wang Shuguang $^{\mathrm{a}}$, Zhu Jincai ${ }^{\mathrm{b}}$, Yang Jinchao ${ }^{\mathrm{c}}$, Chen Jianhua $^{\mathrm{d}}$, Yang Wei $^{\mathrm{e}}$ \\ Northwest Institute of Nuclear Technology, Shaanxi, Xi'an, 710024, China \\ ahitwsg@163.com, bjc_1018@163.com, '1677078841@qq.com, \\ dw941086910@126.com, eyangwei_1018@163.com
}

Keywords: Permeability; Mechanical Analysis; Darcy

\begin{abstract}
Focused on a single end granite tunnel, the feasibility of the assessment method of the average permeability of wall rock based on the Darcy's law has been explored in this paper. In according with the Darcy seepage phenomenon of low-pressure driving gas in porous medium, under the pressurized ventilation, the radon concentration can reduce to the level of winter by summer, so as to determine the critical point of pressurization. According to the air volume variation and other data in the tunnel section, the scope of the average permeability of wall rock can be determined. This method is suitable for the rapid assessment of the average permeability of the wall rock around the underground tunnel with complex structure, and can effectively avoid the macroscopic lack of rock mass permeability measured by the traditional laboratory ratio test of rock samples and in-situ water / gas injection in borehole.
\end{abstract}

\section{Introduction}

Over-broken rock mass belongs to a common soil medium and its brokenness forms large block gap and the matrix cracks and pores with good conduction; thus, it has a higher permeability about one to several orders of magnitude higher than the full and dense rock in the permeability coefficient. Therefore, there is a wide range of engineering application prospect to research the permeability characteristics of over-broken rock mass [1].

In recent years, a lot of achievements of experimental studies on over-broken rock mass seepage had been made: Liu Weiqun [2] and other scholars measured the permeability coefficient variation of sandstone, shale, coal and other over-broken particle rock samples during compaction process with steady-state permeation; With self-designed test equipments and methods, Yu Wei [3] and others worked out the gas permeability of the over-broken sandstone with different grain diameter through the seepage test of gas in over-broken rock mass, and analyzed the permeability variation of over-broken rock mass; With a self-designed test device of gas seepage in loose fragmented medium, Li Guangyue [4] and others carried out an experimental study on the permeability rule of gas leaching from the uranium heap of mining field after in-situ blasting, establishing the ANFIS model to predict permeability; Through the ratio test simulation, Du Yong [5] studied the permeability characteristics of weathering over-broken rock mass. In addition, the crack measurement method and water (gas) injection test in borehole are also commonly used to the study of in-situ permeability characteristics of rock mass [5].

Due to the complex internal structure of rock mass, the laboratory ratio test of rock samples is difficult to reflect the internal structure of real rock mass, and there is a big limitation in test results; and because of the small measuring scale, the currently used in-situ measurements have no macroscopic in their results. Since the underground engineering has the characteristics of big space, wide area and many joints of wall rock and the anisotropic permeability of wall rock, this paper intends to explore a method used for assessing the average permeability of the wall rock around 'underground tunnel with complex structure', so as to compensate deficiencies in existing methods. 


\section{The permeability performance of wall rock around tunnel}

A horizontal single end tunnel is located inside a granite mountain in northwest China, and its axis is laid according to the trend of mountain, with a total length of about 1530m; its buried depth tends to be uniform, with an average of about $200 \mathrm{~m}$. Since there is the radon matrix - uranium in the granite, the radon concentration is perennial higher in the tunnel, and it has obvious seasonal variation: high in summer (about $7.5 \times 10^{4} \mathrm{~Bq} / \mathrm{m}^{3}$ ), low in winter (usually less than $300 \mathrm{~Bq} / \mathrm{m}^{3}$ ), and mediate in spring and autumn. Meanwhile, in the absence of mechanical ventilation, the natural ventilation is perennially existing in the tunnel, and there are the following rules: the air flow changes with the fluctuation of temperature change outside the tunnel, the greater the temperature difference between the tunnel inside and outside, the larger the air flow is; there is significant seasonal characteristics in wind direction changes, reversing for upper and lower layer (see Figure 1). Figure 1 describes qualitatively the natural ventilation within the tunnel in summer and winter: in summer, above the interface (namely the illusion interface with zero speed of natural wind, where the wind starts to reverse), the wind direction is inward, but outward along the tunnel below the interface; it is just the opposite in winter.

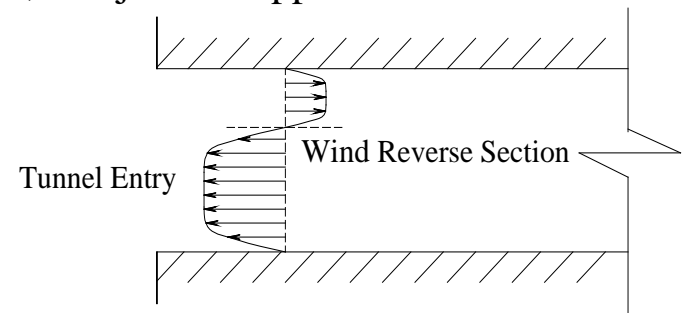

(a) Summer Conditions

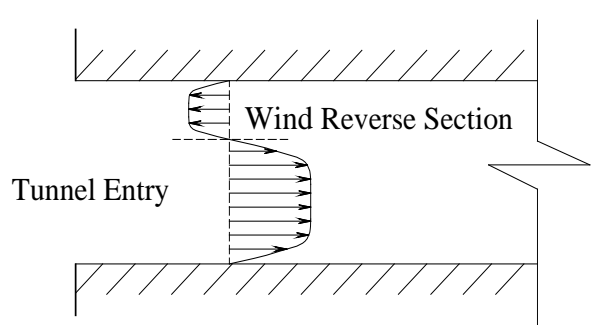

(b) Winter Conditions

Fig. 1 Seasonal change law of natural ventilation in tunnel

It is believed that the change characteristics of radon concentration in the tunnel are mainly caused by the seepage direction changes of gas in the wall rock around the tunnel: in summer, there is the sustained outflow of natural airflow in the tunnel, which flows from the wall rock and carries with a large amount of radon, leading high radon concentration in the tunnel; in winter, there is also the natural airflow, but the opposite direction with summer, that is, the air flows into the tunnel from the outside, and then seeps to mountain outside after getting through the cracks in the wall rock, and during this process, the fresh air outside continues to enter into the wall rock with radon and then exhaust to the mountain outside, so that the radon concentration in tunnel is maintained at a lower level.

Since it is the single end tunnel, without considering the permeability of the mountain, according to the natural ventilation shown in Figure 1, the outflow air in the upper tunnel should be replenished by the reverse airflow from the lower to achieve a balance. To achieve the balance, even if the ventilated section area of tunnel is narrowed, the reverse phenomenon for upper and lower layer is bound to exist, and the interface should be in the middle position of ventilated section, but the reality is not like this.

Set a retaining wall in the tunnel (see Figure 2), and control the shape and size of the ventilated section area by adjusting the numbers of inserting door sheets on the retaining wall. It is found in the experimental measurements, when the ventilated section area is reduced to a certain extent, the natural wind changes to single direction: outward along with the tunnel in summer and inward in winter. This result suggests that the natural wind, from a global point of view, can constantly outflow from the tunnel in summer; in winter, the phenomenon of air inflowing from tunnel outside persists. Obviously, the air flowing outward in summer needs to be replenished by the air flowing inward, and the air flowing inward will flow outside in winter, so as to achieve a natural balance of pressure. Natural ventilation phenomenon in the tunnel indicates that the air seepage can occur in the mountain under natural pressure: flowing into the tunnel from mountain in summer and just the opposite in winter. 

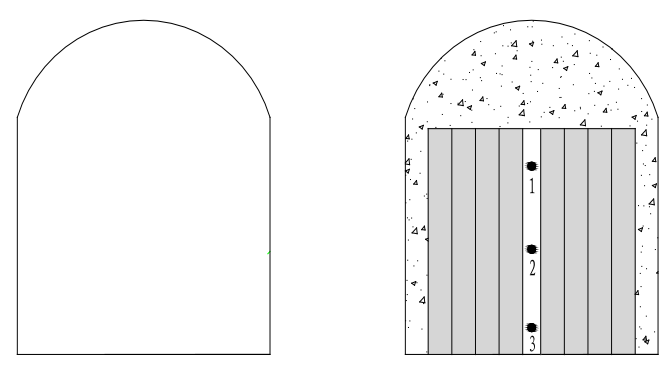

(a) Section of original tunnel (b) rectification section and measurement points

Figure 2 Layout of recitation section and measurement point of tunnel ventilation

Under the natural ventilation, the variation of radon concentration in the tunnel describes that the wall rock around the tunnel here has typical permeability characteristics.

\section{Assessment of the average permeability of wall rock around the tunnel}

Rock mass medium forms a large number of irregular cracks, joints and other intermittent structures under the long time geophysical physical and chemical reactions, so that the rock mass medium shows certain permeability characteristics, which performs as the fluid can get through the cracks or pores. The permeability is one of the physical properties to describe the permeability characteristics of rock mass.

\section{Theoretical basis and formula derivation}

According to Darcy's law, the seepage velocity is directly proportional to pressure gradient, that is, for the same porous medium, different pressure gradients are corresponding to the different seepage velocity. If the pressure gradient and the corresponding seepage velocity within the porous medium can be measured, the permeability of the porous medium can be determined.

Conduct micro-positive pressure aeration for a closed tunnel in the rock mass by using ventilation systems, so that the gas can flow into the wall rock mass from the tunnel. After a sufficiently long time, the pressure field of gas inside the rock mass is stable. The aeration amount within the tunnel is measurable. When the pressure field of gas inside the rock mass is stable, the pressure inside the tunnel is also stable, and the pressure value can be obtained by measurement. According to these two data, and using the formula based on Darcy's law, the permeability, or the average permeability, of rock mass can be worked out.

Now, according to Darcy's law, derive the formula for permeability. First, give some assumptions:

a. the gas is in line with the ideal gas equation of state during the experiment;

b. consider the experiment process as the isothermal process, that is, the density of gas is only related to pressure, and the dynamic viscosity coefficient of gas is constant;

c. ignore the force of gravity;

d. consider the gas flow as laminar flow, namely, the seepage within the medium is in line with Darcy's law;

e. the wall rock is treated as homogeneous porous medium.

Under steady state, one-dimensional Darcy seepage satisfies the mass conservation equation:

$$
\nabla \cdot(\rho v)=0
$$

Wherein, $\rho$ is the gas density, which can be denoted with the ideal gas equation of state: $p=\rho R T ; v$ is the Darcy seepage rate, and the calculation formula is: $v=-\frac{k}{\mu} \nabla p$.

The rock mass permeability $\mathrm{k}$ is the constant, therefore, the equation (1) can be turned into:

$$
\nabla \cdot(p \nabla p)=\Delta p^{2}=0
$$


Turn it into an equation under one-dimensional cylindrical coordinates:

$$
\frac{1}{r} \frac{\partial}{\partial r}\left(r \frac{\partial p^{2}}{\partial r}\right)=0
$$

We can get:

$$
\begin{gathered}
r \frac{\partial p^{2}}{\partial r}=c_{1} \\
p^{2}=\ln r^{c_{1}}+c_{2}
\end{gathered}
$$

Set the equivalent radius of the experimental tunnel as rin, and regard the tunnel with uneven segments of section as a round hole with first-class diameter, and then it's given

$r_{i n}=A / 2 \pi L_{0}$

Wherein, A is considered as the surface area of the tunnel after overbreak (average linear overbreak amount is $300 \mathrm{~mm}), \mathrm{m}^{2}$, and $\mathrm{L}_{0}$ is the total effective length of the tunnel axis, $\mathrm{m}$.

When the pressure field of gas inside the rock mass is stable, the pressure inside the tunnel is $\mathrm{P}_{1}$; at the place $\mathrm{r}_{\text {out }}$ away from the center of the tunnel, assume that the pressure is the initial pressure inside the rock $\mathrm{P}_{0}$, then the corresponding boundary condition is:

We can get:

$$
\left\{\begin{array}{l}
\left.p\right|_{r=r_{\text {in }}}=p_{1} \\
\left.p\right|_{r=r_{\text {out }}}=p_{0}
\end{array}\right.
$$

$$
c_{1}=\left(\ln \frac{r_{\text {in }}}{r_{\text {out }}}\right)^{-1}\left(p_{1}^{2}-p_{0}^{2}\right)
$$

The corresponding $\mathrm{C}_{2}$ can be worked out. During the radial flow of pore medium, the gas pressure gradient is not constant, that is, the gas velocity is not constant.

On the section with the radius of $\mathrm{r}$, the mass flow rate of the gas $(\mathrm{kg} / \mathrm{s})$ is equal, and then according to the ideal gas equation of state, the following formula can be given:

$$
p Q=p_{s c} Q_{s c}=\text { const }
$$

The gas volume flow rate on the section with the radius of $\mathrm{r}$ is $Q=2 \pi r L_{0} v$, wherein, $L_{0}$ is the length of seepage cylindrical surface, that is the effective length of experimental tunnel. It can be given:

$$
\begin{aligned}
p Q & =-2 \pi r L_{0} p \frac{k}{\mu} \frac{\partial p}{\partial r} \\
& =-2 \pi L_{0} \frac{k}{\mu} r p \frac{\partial p}{\partial r} \\
& =-\pi L_{0} \frac{k}{\mu} r \frac{\partial p^{2}}{\partial r}
\end{aligned}
$$

Substitute Formula (4) and (7) into formula (9), there is:

$$
\begin{aligned}
p Q & =-\pi L_{0} \frac{k}{\mu} r \frac{\partial p^{2}}{\partial r} \\
& =-\pi L_{0} \frac{k}{\mu}\left(\ln \frac{r_{\text {in }}}{r_{\text {out }}}\right)^{-1}\left(p_{1}^{2}-p_{0}^{2}\right) \\
& =\pi L_{0} \frac{k}{\mu}\left(\ln \frac{r_{\text {out }}}{r_{\text {in }}}\right)^{-1}\left(p_{1}^{2}-p_{0}^{2}\right)
\end{aligned}
$$

Merge formula (8) and (10), the calculation formula of the permeability of wall rock is:

$$
k=\frac{p_{s c} Q_{s c} \mu \ln \frac{r_{\text {out }}}{r_{\text {in }}}}{\pi L_{\mathrm{o}}\left(p_{1}^{2}-p_{\mathrm{o}}^{2}\right)}
$$

Wherein: $k$ is the average permeability of wall rock around the tunnel, $\mathrm{m}^{2} ; p_{S C}$ is standard atmospheric pressure value, $1.01 \times 10^{5} \mathrm{~Pa} ; Q_{S C}$ is the standard volume flow rate of gas, obtained by 
converting the equation of state, $\mathrm{m}^{3} / \mathrm{s} ; \mu$ is the kinematic viscosity coefficient of air, $1.8 \times 10^{-5} \mathrm{~Pa} \cdot \mathrm{s}$; $r_{\text {in }}$ is the equivalent radius of experimental tunnel, m;Considering that the transfer of gas in fractured rock mass is mainly controlled by seepage and diffusion, $r_{\text {out }}$ is the radius of effective experimental range (define the distance from the tunnel center to the balance point of radon concentration in rock mass), $\mathrm{m}$; $p_{0}$ is the pressure inside the tunnel before aeration, $\mathrm{Pa} ; p_{1}$ is the pressure within the tunnel when the aeration is stable, and the value is the critical aeration pressure (determined according to the following experiments), Pa.

Formula (11) is the permeability calculation formula used in the experiments. According to the formula, the most difficult parameters to determine are the critical aeration pressure $p_{1}$ and the radius of effective experimental range $r_{\text {out }}$, among which, $r_{\text {out }}(7 \sim 10 \mathrm{~m})$ can be valued based on numerical simulation and documentation, and the value of $p_{1}$ should be determined by the following experiments.

\section{Confirmation of the critical aeration pressure}

Power of gas seepage in the rock mass stems from the pressure difference of both sides of the medium. When the aeration pressure aerated to the entire tunnel reaches a certain value, the gas will enter into the wall rock from the tunnel by seepage effect, thus to inhibit the exhalation of radon from the wall rock. Therefore, the radon levels in the tunnel will become the basis to confirm critical aeration pressure (the minimum pressure that reduces the radon concentration in the tunnel to the level of winter).

\section{Pressurized ventilation experiment}

Assume that the gas seepage occurred during the experiment is in line with Darcy's law. Conduct the experiment in summer. According to the sensitivity of radon concentration on the airflow exchange, when the radon concentration in experimental tunnel reaches the level in winter, it is believed that the seepage of the gas in rock mass has the same direction with the winter.

If the tunnel is regarded as a huge hole stretching inside the mountain, and when aerating to the hole to form a certain positive pressure, a part of the gas will discharge through the channel in the mountain with seepage method. During the pressurization process, according to the changes of radon concentration in the tunnel, the airflow exchange between the runnel and wall rock can be determined.

As shown in Figure 3, set an airtight door at the experimental tunnel mouth, and set the ventilation measuring section at the place $150 \mathrm{~m}$ away from tunnel mouth. At the beginning of the experiment, turn off the airtight door at the tunnel mouth, and then open the fans in the two fan rooms to conduct blowing ventilation, adjusting the aeration pressure in tunnel by changing the opening of the airtight door. Respectively measure the ventilation data of airtight door in open and close state (the distribution of wind speed measurement of the section is shown in Figure 4) and obtain three sets of data for each state to get the average results. At the same time, measure the radon concentration at ventilation measuring section.

It is found that, after the aeration pressure reaching 200Pa, the radon concentration in the tunnel can be maintained in the winter level (about $300 \mathrm{~Bq} / \mathrm{m}^{3}$ ), that is, the critical aeration pressure $p_{1}$ can be taken as $200 \mathrm{~Pa}$. Since the designed surplus amount of the wind pressure of fans in the tunnel is more than $500 \mathrm{~Pa}$, it can be considered that the resistance formed in the pressurized ventilation experiment conducted for the tunnel will not affect the air volume in the tunnel sent by fans.

The results of wind speed measurement are shown in Table 1. After closing the airtight door at the tunnel mouth, the air volume of the ventilation system decreases by $12.5 \%$. It can be considered: the air volume lost by system is the air volume escaped to the atmosphere through the mountain by seepage method, and the amount is the air volume difference before and after closing the airtight door at tunnel mouth $\left(1.13 \mathrm{~m}^{3} / \mathrm{s}\right)$. Whereby, the $Q_{S C}$ in formula (11) can be determined based on the ideal gas equation of state, after calculating, $Q_{S C}=0.96 \mathrm{~m}^{3} / \mathrm{s}$. 


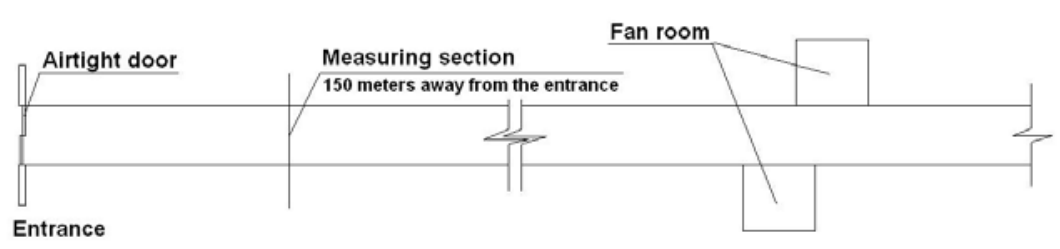

Firgure 3 Layout of experimental tunnel

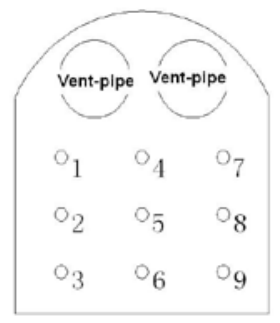

Figure 4 Distribution diagram of wind speed measurement of section

Table 1 Comparison of air volume changes before and after closing the airtight door

\begin{tabular}{crrrrrr}
\hline Items & \multicolumn{7}{c}{ Measurement time $\left(\right.$ July $\left.13^{\text {th }}, 2009\right)$} \\
& $17: 40$ & $17: 45$ & $17: 50$ & $18: 00$ & $18: 05$ & $18: 10$ \\
\hline Average wind speed $(\mathrm{m} / \mathrm{s})$ & 1.53 & 1.56 & 1.54 & 1.37 & 1.33 & 1.36 \\
Air volume $\left(\mathrm{m}^{3} / \mathrm{s}\right)$ & 9.00 & 9.13 & 9.07 & 8.02 & 7.83 & 7.96 \\
Average volume $\left(\mathrm{m}^{3} / \mathrm{s}\right)$ & & 9.07 & & & 7.94 & \\
Air volume difference $\left(\mathrm{m}^{3} / \mathrm{s}\right)$ & \multicolumn{7}{c}{1.13} \\
Rate of change & \multicolumn{7}{c}{ Closed } \\
State of airtight door & \multicolumn{7}{c}{ Opened } \\
\hline
\end{tabular}

Monitoring results of the radon concentration are shown in Figure 5. As can be seen, 2 hours after closing the airtight door, the radon concentration in the tunnel quickly drops to about $300 \mathrm{~Bq} / \mathrm{m}^{3}$ from the average value $7.5 \times 10^{4} \mathrm{~Bq} / \mathrm{m}^{3}$ and remains stable, reaching the level of winter under natural state. Since the radon concentration is very sensitive to airflow exchange, the quickly reduction of radon concentration explains that the exchange direction of airflow between the tunnel and the wall rock has changed. Combined with the seasonal variation of radon concentration, it can be considered that when the seepage direction of the gas in the mountain reverses, that is, the airtight door is closed, the gas inside the tunnel enters into the mountain through the seepage effect under the pressure-driven of 200Pa. Whereby, $p_{1}=200+p_{0}$ in the formula (11) can be determined.

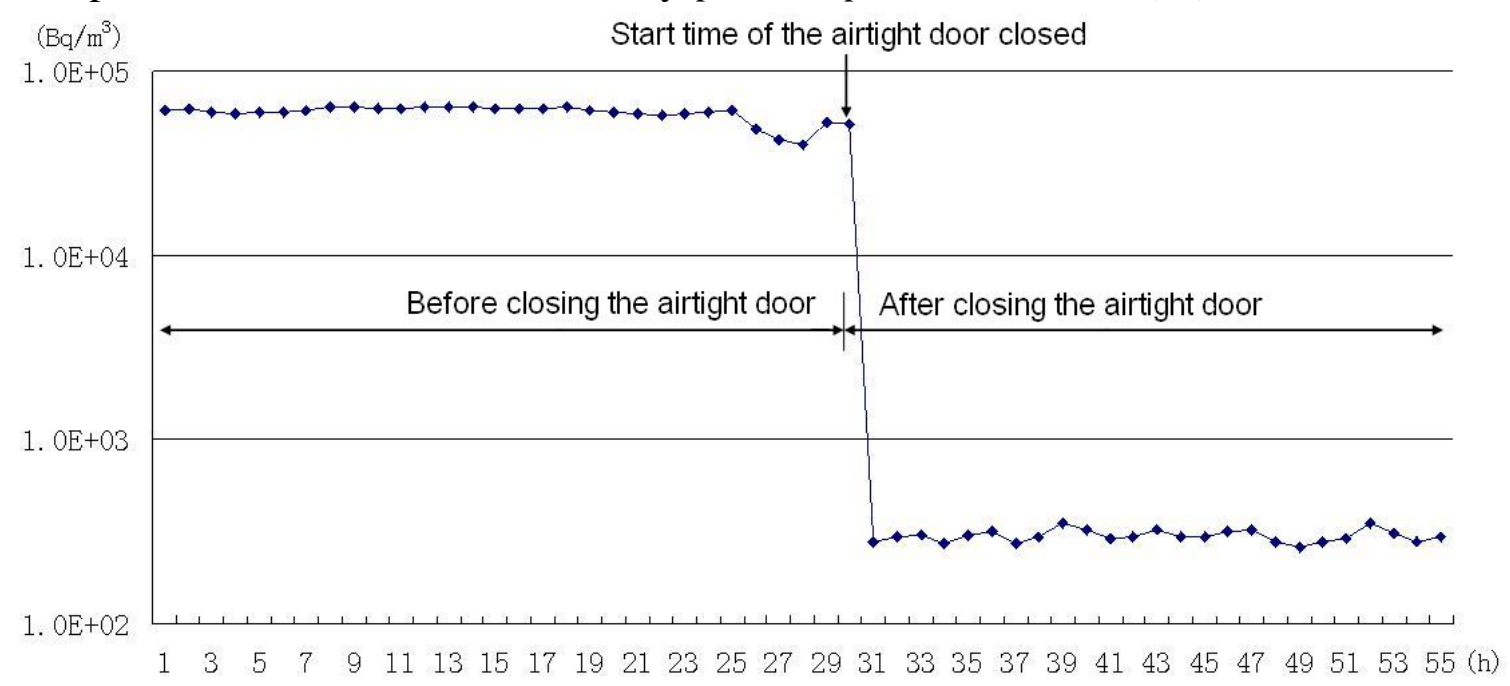

Figure 5 The curve of radon concentration in the tunnel before and after closing the airtight door

\section{Calculation of average permeability of rock mass}

According to the parameters determined by formula (11) and experiments, the average permeability of wall rock around the experimental tunnel can be obtained by calculating: $k$ $=1.5 \times 10^{-11} \sim 1.8 \times 10^{-11} \mathrm{~m}^{2}$. The scope of the granite permeability determined in the literature is $6.1 \times 10^{-12} \sim 9.5 \times 10^{-11} \mathrm{~m}^{2}$, and the result is in line with the definition of the scope. 


\section{Determination of the gas seepage characteristics}

Whether the fluid seepage obeys to Darcy's law can be judged by the seepage Reynolds number. Reynolds number reflects the ratio of inertial and viscous forces when the fluid flows. Numerous studies show that the critical Reynolds number in seepage is $0.2 \sim 0.3$, that is, when the Reynolds number $R e$ is less than or equal to this critical value, the seepage is linear seepage, obeying Darcy's law; when $R e$ is greater than the critical value, the seepage is non-linear seepage, disobeying Darcy's law.

There are many methods to calculate Reynolds number, one generally used at present is:

$$
R e=\frac{v \rho \sqrt{k}}{17.50 \mu \varphi^{\frac{3}{2}}}
$$

Wherein, $R e$ is the Reynolds number; $v$ is the seepage velocity of fluid, $\mathrm{m} / \mathrm{s} ; k$ is the medium permeability, $\mathrm{m}^{2} ; \mu$ is the kinetic viscosity coefficient of the fluid, $\mathrm{Pa} \cdot \mathrm{s} ; \rho$ is the density of the fluid, $\mathrm{kg} / \mathrm{m}^{3} ; \varphi$ is the effective porosity of the medium, \%.;

According to formula (12), to get the seepage Reynolds number, the seepage velocity $v$, the permeability of rock mass medium $k$ and the effective porosity $\varphi$ need to be obtained.

The maximum porosity value of granite is up to $6.11 \%$. Its effective porosity is in the range of $0.05 \% \sim 2.8 \%$, and the effective porosity of general complete bedrock is below $1 \%$. The mountain of studied tunnel has sustained the blasting and other destructive effects during the excavation processes, resulting in the increase of effective porosity. Therefore, the effective porosity of the mountain around the tunnel is calculated by $2 \%$.

According to the experimental results, the seepage velocity calculated by formula $v=Q / A$ is $0.9 \times 10^{-4} \mathrm{~m} / \mathrm{s}$. Substitute the relevant parameters into formula (12), it can be given: $R e=0.0005<0.2$, that is, the gas seepage in the experiment obeys Darcy's law.

Since the foregoing experiment is based on the assumption of Darcy's law, as can be seen from the above analysis results, the assumption is established, justifying the reasonable research process.

\section{Discussions}

In this paper, through the study of seepage characteristics of the gas in the wall rock around an over-broken granite tunnel, a new method for assessing average permeability of the wall rock has been proposed, which can effectively avoid the macroscopic lack of rock mass permeability widely measured by the ratio test of rock samples and in-situ water / gas injection in borehole, broadening the application scope of in-situ permeability measurement technology of the wall rock.

As can be seen from the experiments, using mechanical ventilation to pressurize the tunnel can reduce the radon concentration to the winter level of $300 \mathrm{~Bq} / \mathrm{m}^{3}$ from the summer level of $7.5 \times 10^{4} \mathrm{~Bq} / \mathrm{m}^{3}$ within $1.5 \mathrm{~h}$ and maintain stability. The change of radon concentration suggests that the pressurized ventilation has changed the transfer method of radon in the tunnel. It is believed that the flow method of radon changes into diffusion-driven under pressurized ventilation condition from the seepage domination under natural condition in summer, making the radon transferring into the tunnel from the wall rock. In an indication of the radon concentration, pressurized ventilation achieves the effect to suppress the seepage of radon in wall rock, and even reverses the seepage of radon within the range of shallow depths of the wall rock.

Ventilation method can be extended to other types of wall rock around the tunnel. In the experiments in this paper, the radon concentration change is regarded as the basis to judge the critical pressure of pressurized ventilation. Most wall rocks can not release radon, other tracer gas (e.g.: SF6, etc.) can be used as an indicator to determine the critical pressure. However, this is an experimental method, and there are still limitations. There is no soil and vegetation cover on the surface of the wall rock around the top of this experimental tunnel, and this experimental tunnel is located in the dry region with less rainfall, so that the permeability of the wall rock is higher and the critical pressure of pressurized ventilation is only 200Pa. The smaller the permeability of wall rock, the higher the critical pressure requires. For example, when the surface of the wall rock around the 
tunnel is covered with thick moist soil, the critical pressure needs to be increased. To improve the critical pressure, on the one hand, the airtightness of the tunnel needs to be improved, higher requirement for the project; on the other hand, affected by the pressurized resistance, the ventilation will alter the operating condition of fans before and after pressurizing, and the change because of which should be considered in ventilation rate data.

\section{References}

[1] Wilkening,M.H.Watkins,D.E.Air exchange and 222Rn concentrations in the Carlsbad caverns[J]. Health Physics. 1976,31:139-145

[2] Frédeéric Perrier,Patrick Richon,Umesh Gautam,et al. Seasonal variations of natural ventilation and radon-222 exhalation in a slightly rising dead-end tunnel[J]. Journal of Environmental Radioactivity.2007,97:220-235

[3] Perrier,F.,Richon,P.,Crouzeix,C.,et al.Radon-222 signatures of natural ventilation regimes in an underground quarry[J].Journal of Environmental Radioactivity.2004.71:17-32

[4] Perrier,F.,Richon,P.,Crouzeix,C.,et al.Corrigendum to "Radon-222 signatures of natural ventilation regimes in an underground quarry [Journal of Environmental Radioactivity 71 (2004) 17e32]”[J]. Journal of Environmental Radioactivity.2004.72:369-370

[5] Tanahara,A.,Taira,H.,Takemura,M.Radon distribution and the ventilation of a limestone cave on Okinawa[J]. Geochemical Journal. 1997.31: 49-56

[6] HU Yun jin, SU Bao yu, ZHAN Mei li. Review of the Research on Unsaturated Seepage Flow in Fractured Rock Masses[J]. Journal of Hohai University. 2000.1:40-46

[7] NIU Guanyi,CHEN Jianjie,WANG Jun. Mechanical Analysis of Radon Transport in Vicinity of an Underground Tunnel, Chinese Journal of Computational Physics.2012.2:239-244

[8] ZHANG Jianguo,DU Dianfa,HOU Jian. Oil and gas seepage mechanics(the second edition) [M].DongYing of ShanDong province, China University of Petroleum press. 2010,22-29

[9] Touloukian, Y.S, Jadde W.R. The physical properties of rocks and minerals [M].1990,41-49

[10] ZHAN Zhe. Underground radon exhalation [M].HengYang of HuNan province: Sixth Institute of nuclear industry,1978,211-216 